\title{
Efficient coupling of finite elements and boundary elements - adaptive procedures and preconditioners
}

Ernst P. Stephan ${ }^{1}$

(Received 30 August 2008; revised 23 January 2009)

Dedicated to Professor Ian H. Sloan on the occasion of his 70th birthday.

\section{Abstract}

The article is split into four parts. First, we present the symmetric finite element/boundary element-coupling method. Second, we address the choices of appropriate preconditioners for the resulting discrete system when h- and p-versions are performed. Third, we discuss contact problems which are reduced to variational inequalities. Finally, we show the practical applicability of the finite element/boundary element-coupling method by applying it to a metal turning process. Here the viscoplastic work piece is modelled with finite elements and the linear elastic work tool (milling cutter) is modelled with boundary elements. This leads to an efficient and fast numerical method to simulate the metal turning process and to predict failure of the thermal shrink fit which holds the milling cutter.

http://anziamj . austms.org.au/ojs/index.php/ANZIAMJ/article/view/1495 gives this article, (c) Austral. Mathematical Soc. 2009. Published February 20, 2009. ISSN 1446-8735. (Print two pages per sheet of paper.) 


\section{Contents}

1 Introduction

C775

2 Symmetric FE/BE-coupling

C776

3 Preconditioners for FE/BE-coupling

C783

4 Contact problems with Tresca friction

C787

5 FE/BE for viscoplastic thermo-mechanical coupling

C791

6 Conclusion

C794

References

C795

\section{Introduction}

For a model non-linear transmission problem we present in Section 2 a combined approach with finite elements (FE) and boundary elements (BE). We perform the so-called symmetric coupling which renders all boundary conditions on the interface manifold $\Gamma$ to be natural and allows for a non-linear elliptic differential operator in the bounded domain $\Omega$. Our solution procedure makes use of an integral equation method for the exterior problem and of an energy (variational) method for the interior problems, and consists of coupling both methods via the transmission conditions on the interface. We solve the resulting symmetric coupling formulation with the Galerkin method using finite elements in $\Omega$ and boundary elements on $\Gamma$. We present in Theorem 2 hierarchical error estimators for the FE/BE-coupling and give corresponding numerical results in Table 1 and Figure 1. More details and mixed FE/BE-coupling methods are described by Stephan [24]. Section 3 comments on the solvers and preconditioners for the resulting discrete systems of the FE/BE-coupling. Here, additive Schwarz preconditioners play an 
important role. Then Section 4 considers contact problems with Tresca friction and applies penalty or mortar methods to solve the governing variational inequalities. Then we present error estimates and corresponding adaptive numerical experiments for h- and p-versions. Finally, in Section 5 we apply the $\mathrm{FE} / \mathrm{BE}$-coupling to a viscoplastic thermo-mechanical problem describing a metal forming process [9]. Here the viscoplastic work piece is modelled with finite elements and the linear elastic work tool (milling cutter) is modelled with boundary elements.

\section{Symmetric FE/BE-coupling}

Let $\Omega \subset \mathbb{R}^{\mathrm{d}}, \mathrm{d} \geq 2$, be a bounded domain with Lipschitz boundary $\Gamma=\partial \Omega$ and $\Omega^{\mathrm{c}}=\mathbb{R}^{\mathrm{d}} \backslash \bar{\Omega}$ with normal $\mathrm{n}$ on $\Gamma$ pointing into $\Omega^{\mathrm{c}}$. For given $f \in \mathrm{L}^{2}(\Omega)$, $u_{0} \in H^{1 / 2}(\Gamma), \psi_{0} \in H^{-1 / 2}(\Gamma)$ and $a \in \mathbb{R}$ find $u_{1} \in H^{1}(\Omega)$ and $u_{2} \in H_{\text {loc }}^{1}\left(\Omega^{c}\right)$ such that

$$
\begin{aligned}
-\operatorname{div} A\left(\nabla u_{1}\right) & =f \quad \text { in } \Omega, \\
-\Delta u_{2} & =0 \quad \text { in } \Omega^{c}, \\
u_{1}-u_{2} & =u_{0} \quad \text { on } \Gamma \\
A\left(\nabla u_{1}\right) \cdot \mathfrak{n}-\frac{\partial u_{2}}{\partial n} & =\psi_{0} \quad \text { on } \Gamma, \\
u_{2}(x) & =\left\{\begin{array}{lll}
a \log |x|+o(1), & d=2, \\
O\left(|x|^{-1}\right), & d=3,
\end{array} \quad(|x| \rightarrow \infty) .\right.
\end{aligned}
$$

The operator $A$ is assumed to be uniformly monotone and Lipschitz continuous; operators of this type are considered by Stephan [22] and Zeidler [30]. By using Green's formula together with the decaying condition for $|x| \rightarrow \infty$ in (1) one is led to the representation formula for $\mathfrak{u}_{2}$ in $\Omega^{\mathrm{c}}$

$$
u_{2}(x)=\int_{\Gamma}\left(u_{2}(y) \frac{\partial}{\partial n_{y}} G(x, y)-G(x, y) \frac{\partial u_{2}}{\partial n_{y}}\right) d s_{y}, \quad x \in \Omega^{c},
$$


with the fundamental solution of the Laplacian

$$
G(x, y)= \begin{cases}-\frac{1}{2 \pi} \log |x-y|, & d=2 \\ \frac{1}{4 \pi}|x-y|^{-1}, & d=3\end{cases}
$$

By using the boundary integral operators

$$
\begin{aligned}
V \psi(x) & :=2 \int_{\Gamma} G(x, y) \psi(y) d s_{y}, \quad x \in \Gamma, \\
K \psi(x) & :=2 \int_{\Gamma} \frac{\partial}{\partial n_{y}} G(x, y) \psi(y) d s_{y}, \quad x \in \Gamma, \\
K^{\prime} \psi(x) & :=2 \frac{\partial}{\partial n_{x}} \int_{\Gamma} G(x, y) \psi(y) d s_{y}, \quad x \in \Gamma, \\
W \psi(x) & :=-2 \frac{\partial}{\partial n_{x}} \int_{\Gamma} \frac{\partial}{\partial n_{y}} G(x, y) \psi(y) d s_{y}, \quad x \in \Gamma,
\end{aligned}
$$

together with their well-known jump relations $($ as $x \rightarrow \Gamma$ ) we obtain the integral equations on $\Gamma$

$$
\begin{aligned}
2 \frac{\partial u_{2}}{\partial n} & =-W u_{2}+\left(I-K^{\prime}\right) \frac{\partial u_{2}}{\partial n}, \\
0 & =(I-K) u_{2}+V \frac{\partial u_{2}}{\partial n} .
\end{aligned}
$$

In the interior domain $\Omega$ we apply integration by parts and obtain

$$
\int_{\Omega} A\left(\nabla u_{1}\right) \cdot \nabla v=\int_{\Gamma} A\left(\nabla u_{1}\right) \cdot n v+\int_{\Omega} f v .
$$

Now inserting (8) into (10) and taking the weak form of (9) yield, together with the transmission conditions in (1), the weak form of the transmission problem (1)

$$
\begin{array}{r}
2 \int_{\Omega} A\left(\nabla u_{1}\right) \cdot \nabla v d x-\left\langle\frac{\partial u_{2}}{\partial \mathfrak{n}}, v\right\rangle+\left\langle\mathrm{K}^{\prime} \frac{\partial u_{2}}{\partial \mathfrak{n}}, v\right\rangle+\left\langle W u_{1}, v\right\rangle \\
=2(\mathrm{f}, v)+2\left\langle\psi_{0}, v\right\rangle+\left\langle W u_{0}, v\right\rangle
\end{array}
$$




$$
-\left\langle u_{1}, \psi\right\rangle-\left\langle V \frac{\partial u_{2}}{\partial n}, \psi\right\rangle+\left\langle K u_{1}, \psi\right\rangle=-\left\langle u_{0}, \psi\right\rangle+\left\langle K u_{0}, \psi\right\rangle .
$$

Here we use the inner products

$$
\left\langle u_{1}, \psi\right\rangle=\int_{\Gamma} u_{1} \psi, \quad(f, v)=\int_{\Omega} f v .
$$

In short (11) and (12) read: find $\mathfrak{u}=\mathfrak{u}_{1} \in \mathrm{H}^{1}(\Omega)$ and $\phi=\partial \mathfrak{u}_{2} / \partial \mathrm{n} \in$ $\mathrm{H}^{-1 / 2}(\Gamma)$

$$
\mathcal{A}(\mathrm{u}, \phi ; v, \psi):=2 \int_{\Omega} \mathrm{A}(\nabla \mathrm{u}) \cdot \nabla v \mathrm{~d} x+\mathrm{B}(\mathrm{u}, \phi ; v, \psi)=\mathcal{L}(v, \psi)
$$

for all $v \in \mathrm{H}^{1}(\Omega)$ and $\psi \in \mathrm{H}^{-1 / 2}(\Gamma)$ where $\mathrm{B}(u, \phi ; v, \psi)$ and $\mathcal{L}(v, \psi)$ are defined by the left hand sides and the right hand sides of (11) and (12), respectively.

Remark 1 The above derivation shows that if $\mathfrak{u}_{1}$ and $\boldsymbol{u}_{2}$ solve (1) then $\mathfrak{u}_{1}$ and $\partial u_{2} / \partial n$ satisfy (11) and (12). Conversely, provided $u_{1}$ and $\partial \mathfrak{u}_{2} / \partial n$ solve (11) and (12) then $\boldsymbol{u}_{1}$ and $\boldsymbol{u}_{2}$, defined by (2), are solutions of the transmission problem (1). Furthermore, under the assumptions in (1), the variational formulation is uniquely solvable (as shown by Costabel and Stephan $[8$, 24]).

Let $X_{M}$ and $Y_{N}$ be finite dimensional approximating subspaces of $H^{1}(\Omega)$ and $\mathrm{H}^{-1 / 2}(\Gamma)$, respectively, then the finite element/boundary element Galerkin coupling reads: find $u_{M} \in X_{M}$ and $\phi_{N} \in Y_{N}$ such that

$$
\mathcal{A}\left(\mathfrak{u}_{M}, \phi_{N} ; v, \psi\right):=2 \int_{\Omega} \mathrm{A}\left(\nabla \mathrm{u}_{M}\right) \cdot \nabla v \mathrm{~d} x+\mathrm{B}\left(\mathrm{u}_{M}, \phi_{N} ; v, \psi\right)=\mathcal{L}(v, \psi)
$$

for all $v \in X_{M}$ and $\psi \in Y_{N}$.

As shown by Costabel and Stephan [8, 24] every Galerkin scheme (14) converges with optimal order; that is, with the exact solution of (13) and the Galerkin solution $u_{M}$ and $\phi_{N}$ of (14) there holds $e_{\mathfrak{u}, \phi}:=\left\|\mathfrak{u}-\mathfrak{u}_{M}\right\|_{1, \Omega}+\left\|\phi-\phi_{N}\right\|_{-1 / 2, \Gamma} \lesssim \inf _{\hat{u} \in X_{M}}\|\mathfrak{u}-\hat{u}\|_{1, \Omega}+\inf _{\hat{\phi} \in Y_{N}}\|\phi-\hat{\phi}\|_{-1 / 2, \Gamma}$. 
This gives for the hp-version of the Galerkin scheme (14) on quasiuniform meshes $e_{\mathrm{u}, \phi} \lesssim h^{\alpha} \mathfrak{p}^{-2 \alpha}$ with some $\alpha \in \mathbb{R}$ and on geometrically refined meshes

$$
e_{u, \phi} \lesssim \begin{cases}\exp \left(-b_{1} M^{1 / 3}\right)+\exp \left(-b_{2} N^{1 / 2}\right), & d=2 \\ \exp \left(-b_{1} M^{1 / 5}\right)+\exp \left(-b_{2} N^{1 / 4}\right), & d=3\end{cases}
$$

with some $b_{1}, b_{2} \in \mathbb{R}$ where $M=\operatorname{dof}$ of $X_{M}, N=\operatorname{dof}$ of $Y_{N}$. These results were derived by Guo and Stephan [10], Babuška et al. [2], Maischak and Stephan [16, 23].

Next we restrict ourselves to the $\mathbf{h}$-version of (14), and we introduce a hierarchical error estimator for the FE/BE-coupling. For this purpose we consider regular triangulations $\omega_{H}$ of $\Omega$ and partitions $\gamma_{H}$ of $\Gamma$. Our test and trial spaces are

$$
\begin{aligned}
& \mathrm{T}_{\mathrm{H}}:=\left\{\nu_{\mathrm{H}}: \Omega \rightarrow \mathbb{R} ; \nu_{\mathrm{H}} \text { p.w. linear on } \omega_{\mathrm{H}}, \nu_{\mathrm{H}} \in \mathcal{C}^{0}(\Omega)\right\}, \\
& \tau_{\mathrm{H}}:=\left\{\psi_{\mathrm{H}}: \Gamma \rightarrow \mathbb{R} ; \psi_{\mathrm{H}} \text { p.w. constant on } \gamma_{\mathrm{H}}\right\} .
\end{aligned}
$$

On locally refined meshes we distinguish between old hat functions $b_{\text {old }}$ (being one at the vertices of the triangles) and new hat functions $b_{\text {new }}$ (being one at the midpoints of the edges of the triangles). On the boundary mesh $\gamma_{\mathrm{H}}$ we consider piecewise constant functions $\beta$ with $\beta_{\text {old }}^{i}=1$ on interval $\Gamma_{i}$ and

$$
\beta_{\text {new }}^{i}= \begin{cases}1, & \text { on left half of } \Gamma_{i}, \\ -1, & \text { on right half of } \Gamma_{i} .\end{cases}
$$

Let $n$ denote the number of new nodes, $m$ the number of intervals on the fine boundary mesh and let $\mathrm{T}_{j}=\operatorname{span}\left\{\mathrm{b}_{\text {new }}^{j}\right\}, 1 \leq j \leq n, \tau_{k}=\operatorname{span}\left\{\beta_{\text {new }}^{k}\right\}$, $1 \leq \mathrm{k} \leq \mathrm{m}$. Then one has stable two level subspace decompositions $T_{\mathrm{H} / 2}=$ $\mathrm{T}_{\mathrm{H}} \oplus \mathrm{T}_{1} \oplus \cdots \oplus \mathrm{T}_{\mathrm{n}}, \tau_{\mathrm{H} / 2}=\tau_{\mathrm{H}} \oplus \tau_{1} \oplus \cdots \oplus \tau_{\mathrm{m}}$ of the finite element and boundary element spaces. Now one considers sequences of nested spaces $\tilde{T}_{k} \times \tilde{\tau}_{k} \subset \tilde{T}_{k+1} \times \tilde{\tau}_{k+1}$ starting from $\tilde{T}_{0}=T_{H}, \tilde{T}_{1}=T_{H / 2}, \tilde{\tau}_{0}=\tau_{H}, \tilde{\tau}_{1}=\tau_{H / 2}$. Let $(u, \phi)$ be the exact solution and $\left(\mathfrak{u}_{k}, \phi_{k}\right) \in \tilde{T}_{k} \times \tilde{\tau}_{k}$ be the Galerkin 
solution at level $k \in \mathbb{N}_{0}$. Under the saturation assumption, that is, there exists $\kappa<1$ such that

$$
\left\|\mathfrak{u}-\mathfrak{u}_{k+1}\right\|_{1, \Omega}+\left\|\phi-\phi_{k+1}\right\|_{-1 / 2, \Gamma} \leq \kappa\left(\left\|u-u_{k}\right\|_{1, \Omega}+\left\|\phi-\phi_{k}\right\|_{-1 / 2, \Gamma}\right),
$$

there holds the following theorem. Here and in Table 1 we use the notation

$$
\left\|\mathrm{u}-\mathrm{u}_{\mathrm{L}}, \phi-\phi_{\mathrm{L}}\right\|_{\mathcal{H}}^{2}:=\left\|\mathrm{u}-\mathrm{u}_{\mathrm{k}}\right\|_{1, \Omega}^{2}+\left\|\phi-\phi_{\mathrm{k}}\right\|_{-1 / 2, \Gamma}^{2} .
$$

Theorem 2 (Mund, Stephan [20]) Assuming (17) there holds

$$
\left\|\mathrm{u}-\mathrm{u}_{\mathrm{L}}, \phi-\phi_{\mathrm{L}}\right\|_{\mathcal{H}}^{2} \approx \eta^{2}:=\sum_{i=1}^{n} \Theta_{k, i}^{2}+\sum_{j=1}^{m} \vartheta_{k, j}^{2},
$$

where

$$
\begin{aligned}
\Theta_{k, i} & :=\frac{\left|\mathcal{L}\left(b_{k+1, i}, 0\right)-\mathcal{A}\left(u_{k}, \phi_{k} ;, b_{k+1, i}, 0\right)\right|}{\left\|b_{k+1, i}\right\|_{H^{1}(\Omega)}}, \\
\vartheta_{k, j} & :=\frac{\left|\mathcal{L}\left(0, \beta_{k+1, j}\right)-\mathcal{A}\left(u_{k}, \phi_{k} ; 0, \beta_{k+1, j}\right)\right|}{\left\|\beta_{k+1, j}\right\|_{H^{-1 / 2}(\Gamma)}},
\end{aligned}
$$

with basis functions $\mathrm{b}_{\mathrm{i}, \mathrm{k}+1} \in \tilde{\mathrm{T}}_{\mathrm{k}+1} \backslash \tilde{\mathrm{T}}_{\mathrm{k}}$ and $\beta_{\mathrm{k}+1, \mathrm{j}} \in \tilde{\tau}_{\mathrm{k}+1} \backslash \tilde{\tau}_{\mathrm{k}}$.

Remark 3 A simple adaptive algorithm uses $\Theta_{k, i}$ and $\vartheta_{k, j}$ for local FE/BErefinements and it refines if $\eta_{k}^{r} \geq \theta \max _{1 \leq l \leq N_{k}} \eta_{k}^{l}$ as derived by Mund and Stephan [20], where $\eta_{k}^{r}:=\eta_{k}$ on the rth triangle $\Delta_{k}^{r}$ at level $k, 1 \leq r \leq N_{k}$, and $\theta$ is a preset value.

Remark 4 Residual-type error estimators were derived by Carstensen and Stephan [3] for the h-version of 2D and 3D FE/BE-coupling, namely

$$
\left\|u-u_{h}\right\|_{1, \Omega}+\left\|\phi-\phi_{h}\right\|_{-1 / 2, \Gamma} \leq C\left(R_{1}+R_{2}+R_{3}+R_{4}\right),
$$

where

$$
R_{1}^{2}=\sum_{\Delta \in \omega_{h}} h^{2} \int_{\Delta}|f+\operatorname{div}(\sigma)|^{2} d x
$$




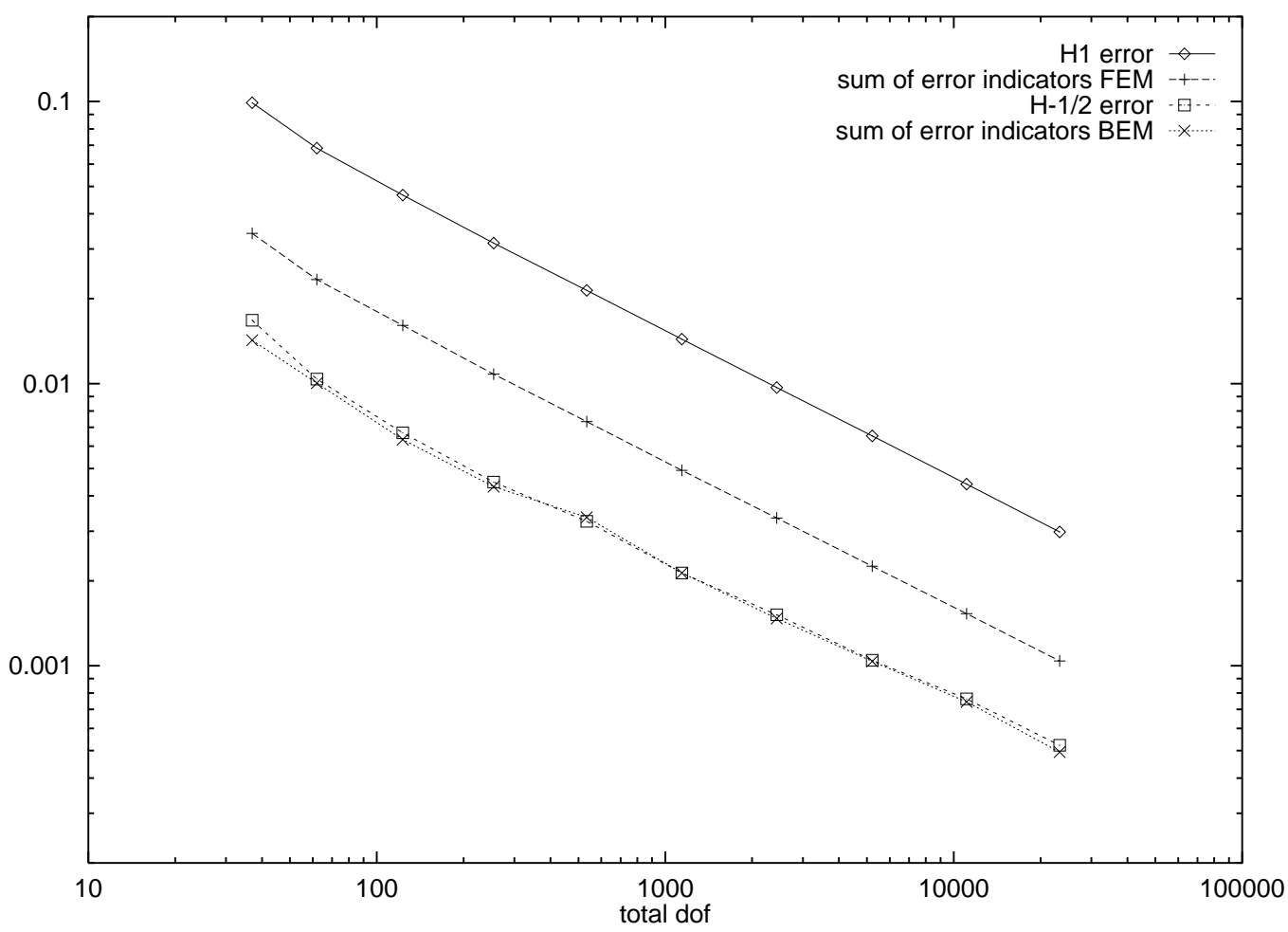

Figure 1: Nonlinear case: error in energy norm/local error indicators [20]. 
TABLE 1: Errors in energy norm $E_{L}$ and error indicator $\eta_{L}$ versus number of unknowns. $\mathrm{L}=$ level, $\mathcal{N}_{\mathrm{L}}=\operatorname{dim} \mathrm{T}_{\mathrm{L}}+\operatorname{dim} \tau_{\mathrm{L}}, \mathrm{E}_{\mathrm{L}}:=\left\|\left(u-\mathfrak{u}_{\mathrm{L}}, \phi-\phi_{\mathrm{L}}\right)\right\|_{\mathcal{H}}$.

\begin{tabular}{rrrcccccc}
\hline $\mathrm{L}$ & $\mathcal{N}_{\mathrm{L}}$ & $\operatorname{dim} \mathrm{T}_{\mathrm{L}}$ & $\operatorname{dim} \tau_{\mathrm{L}}$ & $\mathrm{E}_{\mathrm{L}}$ & $\eta_{\mathrm{L}}$ & $\eta_{\mathrm{L}} / \mathrm{E}_{\mathrm{L}}$ & $\mathrm{K}_{\mathrm{L}}$ & $\alpha_{\mathrm{L}}$ \\
\hline 0 & 37 & 21 & 16 & 0.10040 & 0.03693 & 0.368 & - & - \\
1 & 62 & 44 & 18 & 0.06912 & 0.02546 & 0.368 & 0.688 & 0.505 \\
2 & 123 & 103 & 20 & 0.04708 & 0.01729 & 0.367 & 0.681 & 0.451 \\
3 & 254 & 232 & 22 & 0.03181 & 0.01163 & 0.366 & 0.676 & 0.483 \\
4 & 534 & 510 & 24 & 0.02164 & 0.00807 & 0.373 & 0.680 & 0.489 \\
5 & 1142 & 1112 & 30 & 0.01453 & 0.00537 & 0.369 & 0.671 & 0.511 \\
6 & 2436 & 2400 & 36 & 0.00981 & 0.00365 & 0.372 & 0.675 & 0.511 \\
7 & 5217 & 5175 & 42 & 0.00660 & 0.00248 & 0.375 & 0.673 & 0.515 \\
8 & 11088 & 11036 & 52 & 0.00447 & 0.00170 & 0.381 & 0.676 & 0.516 \\
9 & 23283 & 23217 & 66 & 0.00302 & 0.00115 & 0.381 & 0.677 & 0.525 \\
\hline
\end{tabular}

$$
\begin{aligned}
& R_{2}^{2}=\sum_{\text {interface } E} h_{E} \int_{E}\left|\left[\sigma \cdot h_{E}\right]\right|^{2} d s, \\
& R_{3}^{2}=\sum_{E \text { on } \Gamma} h_{E}\left\|\left\{\sigma \cdot n-\psi_{0}+W\left(u_{h}-v_{0}\right)+\left(K^{\prime}-I\right) \phi_{h}\right\}\right\|_{L^{2}(E)}^{2}, \\
& R_{4}^{2}=\sum_{E \text { on } \Gamma} h_{E}\left\|\frac{\partial}{\partial s}\left((K-I)\left(u_{h}-u_{0}\right)-V \phi_{h}\right)\right\|_{L^{2}(E)}^{2},
\end{aligned}
$$

with $\sigma:=\rho\left(\left|\nabla \mathfrak{u}_{h}\right|\right) \nabla \mathfrak{u}_{h}$.

In numerical experiments reported by Carstensen, Mund and Stephan [20, 3], hierarchical and residual estimators behave similarly, but the computation of $R_{3}$ and $R_{4}$ is expensive. Table 1 lists the error $E_{L}^{2}:=\left\|\mathfrak{u}-u_{L}\right\|_{1, \Omega}^{2}+\| \phi-$ $\phi_{\mathrm{L}} \|_{-1 / 2, \Gamma}^{2}$, the error indicator $\eta_{\mathrm{L}}$, the experimental saturation constant $\kappa_{\mathrm{L}}$, and the experimental convergence rate $\alpha_{\mathrm{L}}$ for problem (1) on an L-shaped domain $\Omega$, when $A\left(\nabla u_{1}\right)=\rho\left(\left|\nabla u_{1}\right|\right) \nabla u_{1}$ with $\rho=\frac{1}{6}\left(1+\frac{5}{1+5 t}\right)$. Figure 1 shows the respective errors in the energy norm and the sums of the local error indicators plotted versus the number of unknowns $\mathcal{N}$. Here $\sum_{i=1}^{n} \Theta_{k, i}^{2}$ is the sum of the error indicators FEM; $\sum_{j=1}^{m} \vartheta_{k, j}^{2}$ is the sum of error indicators 
BEM. Further details are investigated by Mund and Stephan [20].

\section{$3 \quad$ Preconditioners for FE/BE-coupling}

Here we present preconditioners for the hp-version of the symmetric FE/BEcoupling. As iterative solver Heuer, Maischak, Stephan [13] apply the minimum residual method (MINRES); its stable formulation, the hybrid modified conjugate residual method (HMCR), is considered by Mund and Stephan [19]. The linearized Galerkin FE/BE-coupling system (14) is in matrix form

$$
\underbrace{\left(\begin{array}{ccc}
\mathrm{A} & \mathrm{B}^{\top} & 0 \\
\mathrm{~B} & \mathrm{C}+\mathrm{W} & \mathrm{K}^{\top}-\mathrm{I} \\
0 & \mathrm{~K}-\mathrm{I} & -\mathrm{V}
\end{array}\right)}_{=\mathcal{A}}\left(\begin{array}{c}
\mathrm{u}_{\Omega_{1}} \\
\mathrm{u}_{\Gamma} \\
\phi_{\Gamma}
\end{array}\right)=\left(\begin{array}{l}
\mathrm{b}_{1} \\
\mathrm{~b}_{2} \\
\mathrm{~b}_{3}
\end{array}\right)
$$

where the FEM block $A_{N}=\left(\begin{array}{cc}A & B^{T} \\ B & C\end{array}\right)$ correspondents to a Neumann problem for the Laplacian. In (18) W denotes the matrix block belonging to the hypersingular operator and so on; thus we use same letters for matrix and operator. The components $\mathfrak{u}_{\Omega_{1}}, \mathfrak{u}_{\Gamma}$ and $\phi_{\Gamma}$ denote the coefficient vectors of the Galerkin approximations of $u$ in $\Omega_{1}, u$ on $\Gamma$ and $\partial u_{2} / \partial n=\phi$ on $\Gamma$, whereas $b_{i}$ denotes the $i$ th component of the right side in (14).

Considering separately the finite element functions on the interface boundary and in the interior domain, and taking the boundary element functions which discretize the weakly singular operator, we have a splitting of the ansatz space into subspaces. They induce a three-block decomposition of the Galerkin matrix which will be the three-block preconditioner. By this decomposition the strong coupling of edge and interior functions is neglected. Therefore, this three-block splitting allows only for sub-optimal preconditioners. Already in case of exactly inverting the blocks one gets $\mathrm{O}\left(\mathrm{h}^{-3 / 4} \mathrm{p}^{3 / 2}\right)$ iteration numbers. 
In detail we employ a preconditioner of the form

$$
\mathcal{M}_{3}=\left(\begin{array}{ccc}
\tilde{A} & 0 & 0 \\
0 & \tilde{C} & 0 \\
0 & 0 & \tilde{V}
\end{array}\right)
$$

where $\tilde{A}, \tilde{C}$ and $\tilde{V}$ are spectrally equivalent matrices to $A, C$ and $V$, respectively.

Considering the Neumann block as a whole, that is, by taking together finite element functions on the interface and in the interior, we obtain a two-block Jacobi method which has bounded iteration numbers for exact inversion of the two blocks and therefore, allows for almost optimal two-block preconditioners.

Our preconditioning matrix is

$$
\mathcal{M}_{2}=\left(\begin{array}{cc}
\tilde{A}_{M} & 0 \\
0 & \tilde{V}
\end{array}\right)
$$

where $\tilde{A}_{M}$ is spectrally equivalent to $A_{N}+W+M$ and $\tilde{V}$ is spectrally equivalent to $V$. Here $M$ is an additional mass matrix which is added to make $A_{N}+W$ positive definite. As shown by Heuer et al. [13] the iteration numbers of the two-block HMCR are bounded.

The additive Schwarz preconditioner $\mathrm{M}_{\mathrm{ASM}}$ extends the two-block methods by replacing the main blocks by block-diagonal matrices. Here we proceed as follows. First we construct discrete harmonic functions by applying the Schur complement method for the finite element block of the Galerkin matrix. Then, for the finite element part, we decompose the test and trial functions in nodal, edge and interior functions. This amounts to a block Jacobi (Additive Schwarz) preconditioner for the finite element block. We split the boundary element block, belonging to the weakly singular integral operator, into unknowns from a coarse grid space (consisting of piecewise constant functions), and into individual subspaces for each element (consisting of all 
polynomials up to degree $\mathrm{p}$ without the constants). Our preconditioner $M_{\text {diag }}$ is obtained by further splitting the subspaces of edge functions (for both finite elements and boundary elements) into one dimensional subspaces according to the edge basis functions. For the two-block method we obtain in this way two different preconditioned linear systems which need respectively $\mathrm{O}\left(\log ^{2} p\right)$ and $\mathrm{O}\left(\mathrm{p} \log ^{2} p\right)$ minimum residual iterations to be solved up to a given accuracy.

In the first case we apply the Schwarz preconditioner based on decomposing the FE-subspaces $X_{M}$ (as described by Babuška et al. [1]) and the BEsubspace $Y_{N}$ (as described by Tran and Stephan [27]). Here we decompose the FE ansatz space into piecewise constant functions on the boundary mesh and local subspaces on each element spanned by Legendre polynomials. Respective details are given by Heuer et al. [13]. The resulting additive Schwarz preconditioner is called $\mathcal{M}_{\mathrm{ASM}}$. In the second case we take partially diagonal scaling resulting from further refining the subspace decompositions. This block diagonal preconditioner consists of blocks belonging separately to the piecewise linear functions, the interior functions for individual elements and the piecewise constant functions. For the remaining functions we simply take the diagonal of the stiffness matrix $\mathcal{A}$. This method combines the decomposition of $X_{M}$, proposed by Babuška et al. [1], and of $Y_{N}$, proposed by Heuer et al. [15], and gives the preconditioner $\mathcal{M}_{\text {diag. }}$.

Of course we can also apply multilevel preconditioners to the coupled FE/BEsystem of the $h$-version. Here we use multigrid or BPX for the FE matrix $A$ and for the $\mathrm{BE}$ matrices $\mathrm{W}$ and $\mathrm{V}$. Whereas the $\mathrm{FE}$ preconditioners are standard, the BE preconditioners for the boundary integral operators were first analyzed in the concept of multilevel additive Schwarz methods by Tran and Stephan [26]. For a multigrid preconditioner we have bounded iteration numbers of HMCR solvers where for BPX the iteration numbers grow like $\mathcal{O}\left(\log ^{2} \frac{1}{h}\right)$.

Finally we remark that overlapping Schwarz methods for the boundary integral operators (with single layer potential and hypersingular operator) have 
TABLE 2: Numbers of iterations required to reduce residual by a factor of $10^{-3}$ (h-, p-versions) [13].

\begin{tabular}{rrrrrrrr}
\hline $1 / \mathrm{h}$ & $\mathrm{p}$ & $\mathrm{M}+\mathrm{N}$ & $\mathcal{A}$ & $\mathcal{M}_{3}^{-1} \mathcal{A}$ & $\mathcal{M}_{2}^{-1} \mathcal{A}$ & $\mathcal{M}_{\text {ASM }}^{-1} \mathcal{A}$ & $\mathcal{M}_{\text {diag }}^{-1} \mathcal{A}$ \\
\hline 2 & 2 & 37 & 36 & 21 & 11 & 30 & 30 \\
2 & 4 & 97 & 138 & 34 & 12 & 47 & 52 \\
2 & 6 & 181 & 285 & 40 & 13 & 54 & 67 \\
2 & 8 & 289 & 536 & 45 & 13 & 62 & 77 \\
2 & 10 & 421 & 892 & 50 & 13 & 66 & 94 \\
2 & 12 & 577 & 1374 & 55 & 13 & 74 & 111 \\
2 & 14 & 757 & 2328 & 60 & 13 & 82 & 135 \\
2 & 16 & 961 & 9999 & 79 & 17 & 102 & 197 \\
\hline 4 & 1 & 37 & 17 & 15 & 10 & & \\
8 & 1 & 97 & 38 & 23 & 13 & & \\
16 & 1 & 289 & 67 & 32 & 13 & & \\
32 & 1 & 961 & 130 & 45 & 14 & & \\
64 & 1 & 3457 & 243 & 60 & 15 & & \\
128 & 1 & 13057 & 476 & 75 & 15 & & \\
\hline
\end{tabular}


been studied by Tran and Stephan [28] and non-overlapping Schwarz methods by Heuer, Leydecker and Stephan [12, 14].

\section{Contact problems with Tresca friction}

We consider two non-overlapping polygonal domains $\Omega^{i}, i=1,2$, with Lipschitz boundaries $\Gamma^{\mathrm{i}}$. Each $\Gamma^{\mathrm{i}}$ consists of three mutually disjoint measurable parts $\Gamma_{\mathrm{D}}^{\mathrm{i}}, \Gamma_{\mathrm{N}}^{\mathrm{i}}$ and $\Gamma_{\mathrm{C}}^{\mathrm{i}}$, where Dirichlet, Neumann and frictional contact conditions are prescribed. Let $\mathrm{g}(\mathrm{x}), \mathrm{x} \in \Gamma_{\mathrm{C}}:=\Gamma_{\mathrm{C}}^{1} \cup \Gamma_{\mathrm{C}}^{2}$, be the distance between the bodies. We define a jump $h \in \mathcal{C}\left(\Gamma_{\mathrm{C}}\right)$ across $\Gamma_{\mathrm{C}}$ pointwise as $[h](x):=h(x)-h(b(x)), x \in \Gamma_{C}$, with a bijective mapping $b: \Gamma_{C}^{1} \rightarrow \Gamma_{C}^{2}$, where the normal or the tangential displacement $\left(\mathfrak{u}_{\mathfrak{n}}:=\mathbf{u} \cdot \mathfrak{n}\right.$ or $\left.\mathfrak{u}_{\mathbf{t}}:=\mathbf{u} \cdot \mathbf{t}\right)$ is at $h(x)$. The stress tensor $\sigma$ is determined by $\mathbf{u}$ with Hooke's law and under small strain assumptions there holds with $\lambda>0$ and $\mu>0$

$$
\sigma(\mathbf{u})=\lambda \operatorname{tr} \varepsilon(\mathbf{u})+2 \mu \varepsilon(\mathbf{u}), \quad \varepsilon(\mathbf{u})=\left(\nabla \mathbf{u}+\nabla \mathbf{u}^{\top}\right) / 2 .
$$

The two body contact problem reads: for given traction $\hat{\mathbf{t}}$ on $\Gamma_{\mathrm{N}}$, friction $\mathcal{F} \geq 0$, and gap g, find displacement $\mathbf{u}: \Omega=\Omega^{1} \cup \Omega^{2} \rightarrow \mathbb{R}^{3}$ such that

$$
\begin{aligned}
\operatorname{div} \sigma(\mathbf{u})=0 & \text { in } \Omega, \\
\mathbf{u}=0 & \text { on } \Gamma_{\mathrm{D}}:=\Gamma_{\mathrm{D}}^{1} \cup \Gamma_{\mathrm{D}}^{2}, \\
\sigma(\mathbf{u}) \cdot \mathfrak{n}=\hat{\mathbf{t}} & \text { on } \Gamma_{\mathrm{N}}:=\Gamma_{\mathrm{N}}^{1} \cup \Gamma_{\mathrm{N}}^{2}, \\
\left.\begin{array}{rl}
\sigma_{\mathfrak{n}} \leq 0, \quad\left[u_{n}\right] \leq \mathrm{g}, \quad \sigma_{\mathfrak{n}}\left(\left[\mathbf{u}_{\mathrm{n}}\right]-\mathrm{g}\right)=0 \\
\left|\sigma_{\mathrm{t}}\right| \leq \mathcal{F}, \quad \sigma_{\mathrm{t}}\left[\mathrm{u}_{\mathrm{t}}\right]+\mathcal{F}\left|\left[\mathrm{u}_{\mathrm{t}}\right]\right|=0
\end{array}\right\} & \text { on } \Gamma_{\mathrm{C}},
\end{aligned}
$$

with scalar normal and tangential stresses $\sigma_{\mathfrak{n}}:=\mathbf{n} \cdot \sigma(\mathbf{u}) \cdot \mathbf{n}, \sigma_{\mathrm{t}}:=\mathbf{t} \cdot \sigma(\mathbf{u}) \cdot \mathbf{n}$. The given friction function $\mathcal{F}$ defines pointwise the sticking threshold of the bodies; that is, as seen from (21), if the absolute value of the tangential stress does not reach the given friction $\left|\sigma_{t}\right| \leq \mathcal{F}$, then $\left[\mathfrak{u}_{t}\right]=0$, and $\left[\boldsymbol{u}_{t}\right] \neq 0$ is only possible if $\left[\sigma_{t}\right]=\mathcal{F}$. 
As shown by Chernov, Maischak and Stephan $[6,7]$, the two body problem (21) is reduced to the following domain variation inequality for admissible functions belonging to the convex cone $\mathrm{K}_{\Omega}:=\left\{\mathbf{u} \in \mathrm{H}_{\mathrm{D}}^{1}(\Omega)\right.$, $\left[\mathrm{u}_{n}\right] \leq \mathrm{g}$ on $\left.\Gamma_{\mathrm{C}}\right\}$; that is, find $\mathbf{u} \in \mathrm{K}_{\Omega}$ such that

$$
\int_{\Omega} \sigma(\mathbf{u}): \varepsilon(\boldsymbol{v}-\mathbf{u}) \mathrm{d} x+\int_{\Gamma_{\mathrm{C}}} \mathcal{F}\left(\left|\left[v_{\mathrm{t}}\right]\right|-\left|\left[\boldsymbol{u}_{\mathrm{t}}\right]\right|\right) \mathrm{ds} \geq \int_{\Gamma_{\mathrm{N}}} \hat{\mathbf{t}} \cdot(\boldsymbol{v}-\mathbf{u}) \mathrm{ds}
$$

for all $\boldsymbol{v} \in \mathcal{K}_{\Omega}$, where : denotes the standard tensor product (as used by Wriggers [29]). Integration by parts gives

$$
\int_{\Omega} \sigma(\mathbf{u}): \varepsilon(\boldsymbol{v}-\mathbf{u}) \mathrm{d} x=\int_{\Gamma} \mathcal{T}(\mathbf{u}) \cdot(\boldsymbol{v}-\mathbf{u}) \mathrm{d} s-\int_{\Omega} \operatorname{div} \sigma(\mathbf{u}) \cdot(\boldsymbol{v}-\mathbf{u}) \mathrm{d} x
$$

with $\operatorname{div} \sigma(\mathbf{u})=0$ in $\Omega$. On the other hand the DtN-map or Steklov-Poincaré operator $\mathrm{S}=\mathrm{W}+\left(\mathrm{I}+\mathrm{K}^{\prime}\right) \mathrm{V}^{-1}(\mathrm{I}+\mathrm{K})$ satisfies $\langle\mathcal{T}(\mathbf{u}), \boldsymbol{v}-\mathbf{u}\rangle=\langle\mathrm{Su}, \boldsymbol{v}-\mathbf{u}\rangle$ where $\langle\cdot, \cdot\rangle$ means integration on $\Gamma$. Hence using (22) and (23) one reduces problem (21) to the boundary variational inequality: find $\mathbf{u} \in \mathcal{K}:=$ $\left\{\boldsymbol{u} \in \tilde{\mathrm{H}}^{1 / 2}\left(\Gamma \backslash \Gamma_{\mathrm{D}}\right),\left[\boldsymbol{u}_{\mathrm{n}}\right] \leq \mathrm{g}\right.$ on $\left.\Gamma_{\mathrm{C}}\right\}$ such that for all $\boldsymbol{v} \in \mathcal{K}$

$$
\int_{\Gamma}(\mathbf{S u}) \cdot(\boldsymbol{v}-\mathbf{u}) \mathrm{d} s+\int_{\Gamma_{\mathrm{C}}} \mathcal{F}\left(\left|\left[v_{\mathrm{t}}\right]\right|-\left|\left[\boldsymbol{u}_{\mathrm{t}}\right]\right|\right) \mathrm{ds} \geq \int_{\Gamma_{\mathrm{N}}} \hat{\mathbf{t}} \cdot(\boldsymbol{v}-\mathbf{u}) \mathrm{ds}
$$

Applying penalty methods to (22) is a standard approach for FE-simulations of contact problems. As analyzed by Chernov et al. [5] we approximate the boundary variational inequality (24) by the penalty method; that is, we find $\mathbf{u}^{\varepsilon} \in \tilde{\mathrm{H}}^{1 / 2}\left(\Gamma \backslash \Gamma_{\mathrm{D}}\right)$ such that

$\int_{\Gamma} S \mathbf{u}^{\varepsilon} \cdot \boldsymbol{\phi} d s+\int_{\Gamma_{C}} \varepsilon_{n}^{-1}\left[\mathfrak{u}_{n}^{\varepsilon}\right]^{+}\left[\phi_{n}\right] d s+\int_{\Gamma_{C}} \varepsilon_{t}^{-1} \mathcal{F}\left[\mathfrak{u}_{\mathrm{t}}^{\varepsilon}\right]^{*}\left[\phi_{\mathrm{t}}\right] \mathrm{ds}=\int_{\Gamma_{\mathrm{N}}} \hat{\mathbf{t}}_{\mathrm{N}} \cdot \boldsymbol{\phi} \mathrm{ds}$ where $\varepsilon_{n}, \varepsilon_{\mathrm{t}}>0$ are penalty parameters, $a^{*}:=\operatorname{sign}(\mathrm{a}) \min \left(\varepsilon_{\mathrm{t}},|\mathrm{a}|\right), \mathrm{a}^{+}:=$ $\max (a, 0)$ for some $a \in \mathbb{R}$. Note that the penalty method is formulated on the unconstrained space $\tilde{H}\left(\Gamma \backslash \Gamma_{\mathrm{D}}\right)$ and with $\mathrm{g} \equiv 0$ for simplicity. 
Let $\boldsymbol{\psi}^{\varepsilon}=S \mathbf{u}^{\varepsilon}$ be the traction, $\boldsymbol{\Psi}^{\varepsilon}$ its Galerkin approximation with exact $\mathbf{u}^{\varepsilon}$ and its approximation $\mathbf{U}^{\varepsilon}$, and $\mathbf{P}_{\mathfrak{n}}^{\varepsilon}$ and $P_{\mathfrak{t}}^{\varepsilon}$ be the Galerkin approximation of $p_{\mathfrak{n}}^{\varepsilon}$ and $p_{\mathfrak{t}}^{\varepsilon}$ respectively. We define the (triple bar) norm

$$
\begin{aligned}
\| \mid \mathbf{u}^{\varepsilon} & -\mathbf{u}^{\varepsilon} \| \mid:=\left(\left\|\mathbf{u}^{\varepsilon}-\mathbf{u}^{\varepsilon}\right\|_{\mathrm{H}^{1 / 2}(\Gamma)}^{2}+\left\|\boldsymbol{\Psi}^{\varepsilon}-\boldsymbol{\Psi}^{\varepsilon}\right\|_{\mathrm{H}^{-1 / 2}(\Gamma)}^{2}\right. \\
& \left.+\left\|\varepsilon_{\mathrm{n}}^{1 / 2}\left(p_{\mathrm{n}}^{\varepsilon}-\mathrm{P}_{\mathrm{n}}^{\varepsilon}\right)\right\|_{\mathrm{L}^{2}\left(\Gamma_{\mathrm{C}}\right)}^{2}+\left\|\varepsilon_{\mathrm{t}}^{1 / 2} \mathcal{F}^{-1 / 2}\left(p_{\mathrm{t}}^{\varepsilon}-\mathrm{P}_{\mathrm{t}}^{\varepsilon}\right)\right\|_{\mathrm{L}^{2}\left(\Gamma_{\mathrm{C}}\right)}^{2}\right)^{1 / 2} .
\end{aligned}
$$

The following residual a posteriori error estimate is proven by Chernov [4] for the $h$-version of the FE/BE-coupling on quasiuniform meshes $\mathcal{T}_{h}$ if $\varepsilon_{\mathrm{n}} \geq \tilde{\mathrm{C}} \mathrm{h}$ and $\varepsilon_{\mathrm{t}} \geq \tilde{\mathrm{C}} \mathcal{F} \mathrm{h}$ for some constant $\tilde{\mathrm{C}}>0$

$$
c \sum_{\mathrm{I} \in \mathcal{T}_{h}} \eta_{h}^{2}(\mathrm{I}) \leq\left\|\left|\mathbf{u}^{\varepsilon}-\mathbf{u}^{\varepsilon} \|\right|^{2} \leq \mathrm{C} \sum_{\mathrm{I} \in \mathcal{T}_{\mathrm{h}}} \eta_{h}^{2}(\mathrm{I})\right.
$$

with the local error indicators

$$
\begin{aligned}
\eta_{h}^{2}(\mathrm{I}):= & h_{\mathrm{I}}\left\|\hat{\mathfrak{t}}-\hat{S} \mathbf{U}^{\varepsilon}\right\|_{\mathrm{L}^{2}\left(\mathrm{I} \cap \Gamma_{\mathrm{N}}\right)}^{2}+\mathrm{h}_{\mathrm{I}}\left\|P_{\mathfrak{n}}^{\varepsilon} \mathfrak{n}+\mathrm{P}_{\mathrm{t}}^{\varepsilon} \mathbf{t}-\hat{S} \mathbf{U}^{\varepsilon}\right\|_{\mathrm{L}^{2}\left(\mathrm{I} \cap \Gamma_{\mathrm{C}}\right)}^{2} \\
& +\mathrm{h}_{\mathrm{I}}\left\|\frac{\partial}{\partial \mathrm{s}}\left(\mathbf{V} \mathbf{\Psi}^{\varepsilon}-(\mathrm{K}+1 / 2) \mathbf{U}^{\varepsilon}\right)\right\|_{\mathrm{L}^{2}(\mathrm{I})}^{2} .
\end{aligned}
$$

Here $\widehat{S}$ is the discretization of $\mathbf{S}$ (as described by Chernov et al. [5]), I $\in \mathcal{T}_{h}$ is the mesh element and $\mathrm{c}$ and $\mathrm{C}$ are positive constants independent of the mesh size $h$.

Figure 2 shows a frictional contact problem (with Tresca friction) between an elastic body $\Omega=[-1,1]^{2}$ and a rigid obstacle $\gamma:=[-1,1] \times\{-1$, d $\}$ which is pushed upwards with $d=0.6 \cdot 10^{-4}$. Further details were given by Chernov and Stephan [7].

An alternative technique for approximately solving the variational inequality (24) is the mortar method analyzed by Chernov et al. [6]. In contrast to the penalty method, no intermediate formulation is needed and the discrete version of the variational inequality (24) is solved. In order to obtain the hp-version of the BEM for the inequality (24) via the mortar method, 

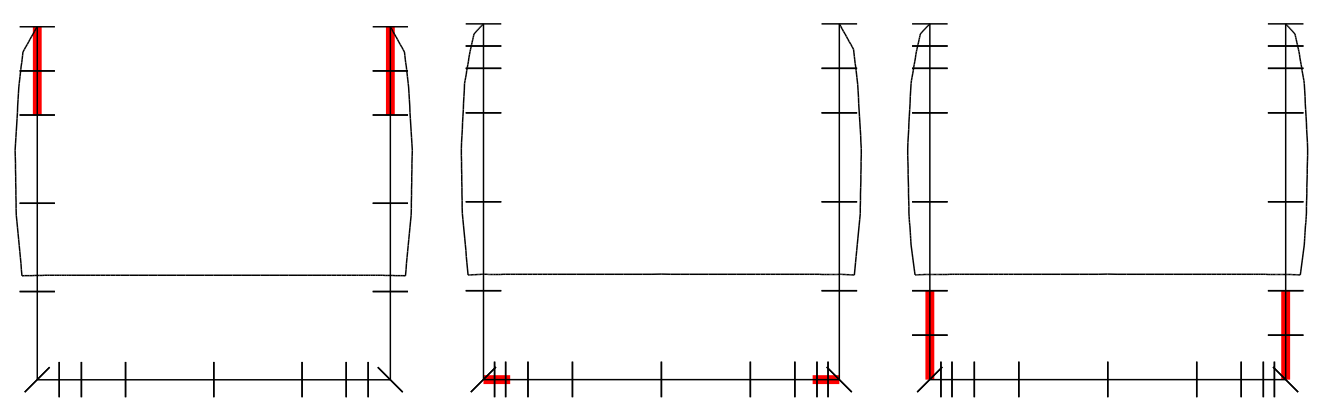

Figure 2: Sequence of the adaptively generated meshes and deformed geometries [7].

we decompose each $\Gamma^{i}, i=1,2$, into a finite family of straight line segments $\mathcal{T}_{h}^{i}$ such that each segment belongs to $\Gamma_{\mathrm{D}}^{\mathrm{i}}$, or $\Gamma_{\mathrm{N}}^{\mathrm{i}}$, or $\Gamma_{\mathrm{C}}^{\mathrm{i}}$. Based on $\mathcal{T}_{\mathrm{h}}^{\mathrm{i}}$, $i=1,2$, we define the space of globally continuous piecewise polynomial functions $\mathcal{V}_{\mathrm{hp}}^{\mathrm{i}}$ (discretization of the displacement) and the space of globally discontinuous piecewise polynomial functions $\mathcal{W}_{\mathrm{hp}}^{\mathrm{i}}$ (discretization of the traction). Let $\mathcal{P}_{\mathrm{p}_{\mathrm{I}}}$ (I) be the space of polynomials on I of degree at most $p_{\mathrm{I}}$. Then we demand $\left.\mathbf{U}\right|_{\mathrm{I}} \in \mathcal{P}_{\mathrm{p}_{\mathrm{I}}}(\mathrm{I})$ and $\left.\boldsymbol{\Psi}\right|_{\mathrm{I}} \in \mathcal{P}_{\mathrm{p}_{\mathrm{I}}-1}(\mathrm{I})$ for arbitrary $\mathbf{U} \in \mathcal{V}_{\mathrm{hp}}^{\mathrm{i}}$ and $\boldsymbol{\Psi} \in \mathcal{W}_{\mathrm{hp}}^{\mathrm{i}}$. Note that the mesh nodes and the polynomial degrees do not match in general across $\Gamma_{\mathrm{C}}$, which is strongly desirable in many applications considered by Wriggers [29]. On the other hand, the non-matching property makes discretization of the convex set of admissible solutions $\mathcal{K}$ in (24) more complex, since the condition $u_{n}^{1}-u_{n}^{2} \equiv\left[u_{n}\right] \leq g$ is difficult to realize in each $\boldsymbol{x} \in \Gamma_{\mathrm{C}}$. We impose this condition in a weak sense. In order to define discrete contact conditions we introduce auxiliary spaces of normal traces on $\Gamma_{\mathrm{C}}^{\mathrm{i}}$ by $\mathcal{N}_{\mathrm{hp}}^{\mathrm{i}}:=\left\{\mathbf{W}=\left.\mathbf{U} \cdot \mathbf{n}^{\mathrm{i}}\right|_{\Gamma_{\mathrm{C}}}: \mathbf{U} \in \mathcal{V}_{\mathrm{hp}}^{\mathrm{i}}\right\}$, and the mortar space $\mathcal{M}_{\mathrm{hp}}^{1}:=\left\{\Psi \in \mathcal{N}_{\mathrm{hp}}^{1}: \Psi \in \mathcal{P}_{\mathrm{p}_{\mathrm{I}}-1}(\mathrm{I})\right.$, if $\left.\mathrm{I} \cap \partial \Gamma_{\mathrm{C}} \neq \emptyset\right\}$, without loss of generality associated with $\mathcal{T}_{h}^{1}$. We define the hp-mortar projection operator (as introduced by Chernov et al. [6] and by Seshaiyer and Suri [21]) 


$$
\begin{aligned}
\pi_{\mathrm{hp}}^{1}: \mathrm{H}^{1 / 2}\left(\Gamma_{\mathrm{C}}\right) \rightarrow & \mathcal{N}_{\mathrm{hp}}^{1} \text { by } \\
& \pi_{\mathrm{hp}}^{1} \phi=\phi \quad \text { in } \partial \Gamma_{\mathrm{C}}, \\
& \int_{\Gamma_{\mathrm{C}}}\left(\phi-\pi_{\mathrm{hp}}^{1} \phi\right) \Psi^{1} \mathrm{ds}=0 \quad \text { for all } \Psi^{1} \in \mathcal{M}_{\mathrm{hp}}^{1} .
\end{aligned}
$$

Let $\mathrm{G}_{\mathrm{hp}}^{i}$ be the set of Gauss-Lobatto nodes associated with the elements of $\mathcal{T}_{\mathrm{h}}^{\mathrm{i}}$. Let $\mathcal{V}_{\mathrm{hp}}:=\mathcal{V}_{\mathrm{hp}}^{1} \times \mathcal{V}_{\mathrm{hp}}^{2}$. Now we give the discrete counterpart of (24) obtained by the hp-version BEM and the mortar projection: find $\mathbf{U} \in \mathcal{K}_{\mathrm{hp}}:=$ $\left\{\mathbf{U} \in \mathcal{V}_{\mathrm{hp}}: \pi_{\mathrm{hp}}^{1}\left[\mathrm{U}_{\mathrm{n}}\right](\boldsymbol{x}) \leq \mathrm{g}(\boldsymbol{x})\right.$ for all $\left.\boldsymbol{x} \in \mathrm{G}_{\mathrm{hp}}^{1} \cap \Gamma_{\mathrm{C}}\right\}$ :

$$
\begin{array}{r}
\int_{\Gamma}(\hat{\mathbf{S}} \mathbf{U}) \cdot(\boldsymbol{\Phi}-\mathbf{U}) \mathrm{d} s+\int_{\Gamma_{\mathrm{C}}} \mathcal{F}\left(\left|\pi_{\mathrm{hp}}^{1}\left[\Phi_{\mathrm{t}}\right]\right|-\left|\pi_{\mathrm{hp}}^{1}\left[\mathrm{U}_{\mathrm{t}}\right]\right|\right) \mathrm{d} s \\
\geq \int_{\Gamma_{\mathrm{N}}} \hat{\mathbf{t}} \cdot(\boldsymbol{\Phi}-\mathbf{u}) \mathrm{d} s \quad \text { for all } \boldsymbol{\Phi} \in \mathcal{K}_{\mathrm{hp}} .
\end{array}
$$

Note that in general $\mathcal{K}_{\text {hp }} \not \subset \mathcal{K}$.

A heuristic local a posteriori error indicator for the variational inequality (28) was presented by Chernov and Stephan [7] together with numerical experiments (based on a three-step hp-adaptive algorithm introduced by Maischak and Stephan [17]). Our experiments [7] (compare Figures 3 and 4) show that this adaptive procedure leads to appropriate mesh refinement and distribution of polynomial degrees (given by the numbers in Figure 4) respecting the singular behavior of the solution at the contact zone and at the corners or where the boundary conditions change.

\section{$5 \quad \mathrm{FE} / \mathrm{BE}$ for viscoplastic thermo-mechanical coupling}

To compute the metal turning process, we use the following FE/BE-procedure for the velocity and temperature formulation of the viscoplastic thermo- 

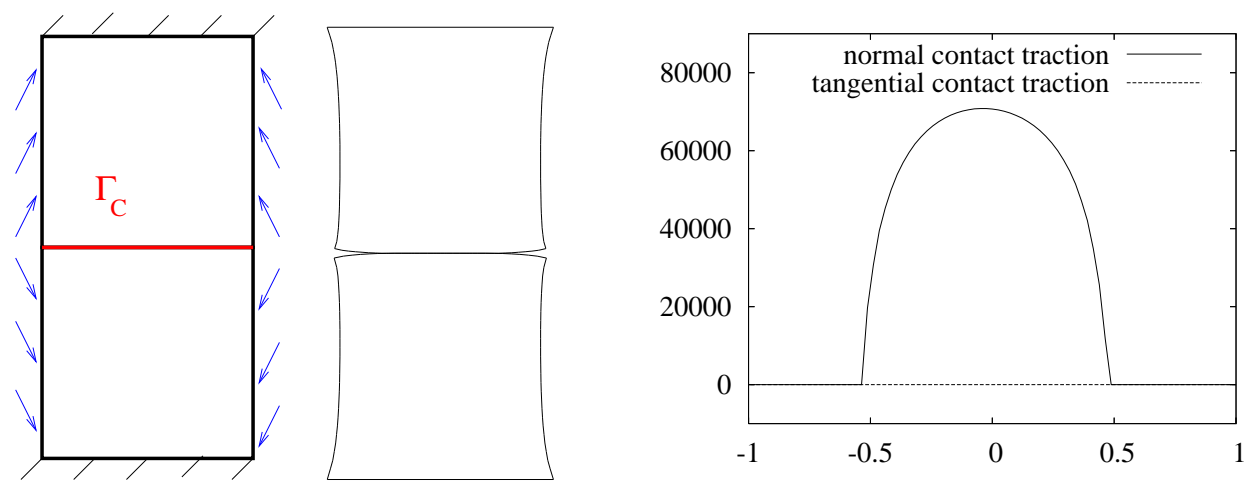

Figure 3: Model problem, deformed configuration and contact traction [7].
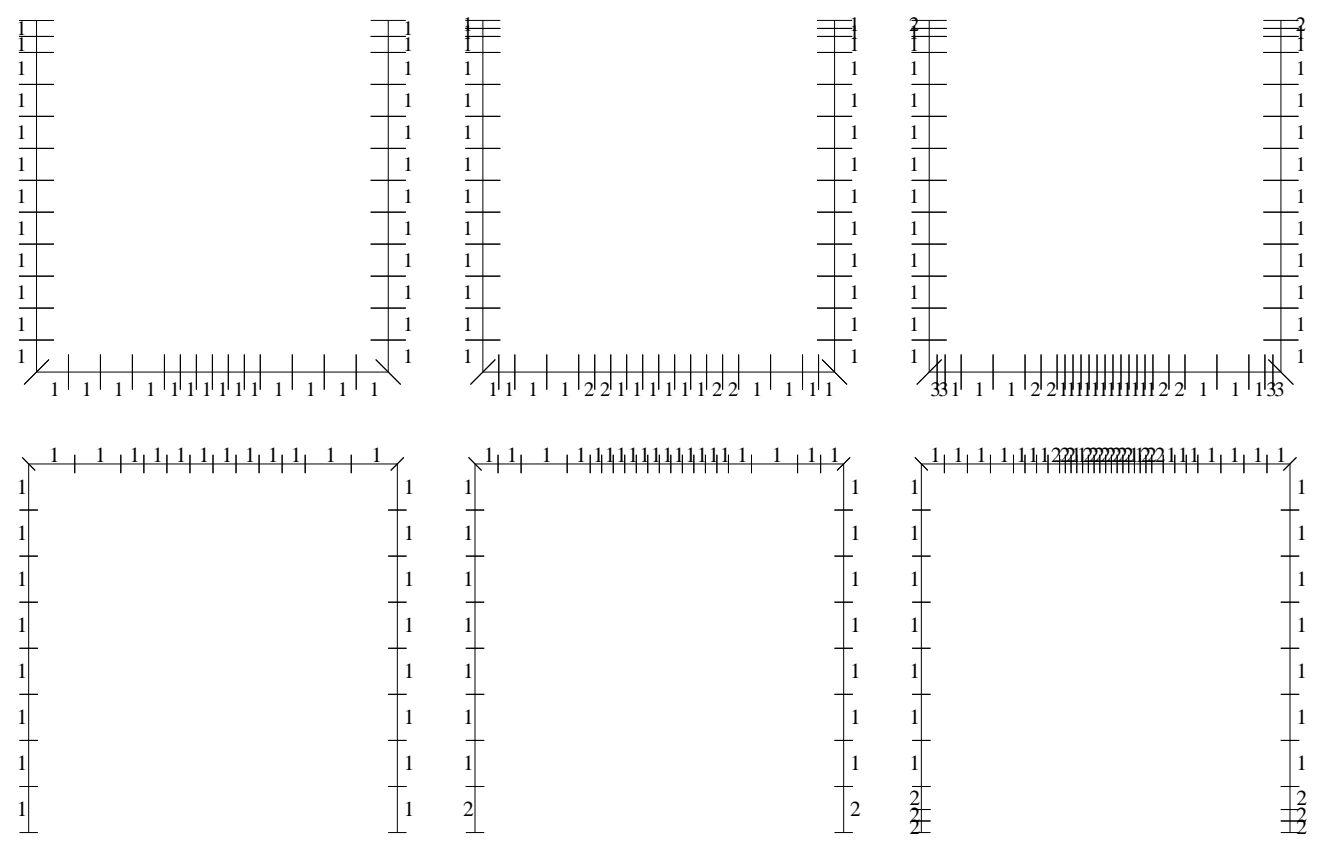

FiguRE 4: Adaptively generated meshes and polynomial degrees after three, six and nine refinement steps [7]. 
mechanical contact problem. For this purpose we choose Hart's constitutive model to allow viscoplastic behaviour in the workpiece. Keeping track of two internal state variables, we only have to solve a series of linear elastic problems using the hypoelastic material law. These variables are the anelastic strain tensor $\dot{\varepsilon}^{a}$ and a scalar $\sigma^{(*)}$, called hardness, which is similar to an isotropic strain hardening parameter or current yield stress. As opposed to other viscoplastic material models, Hart's model enables us to use finite elements as well as boundary elements for a viscoplastic workpiece. Further details are investigated by Stephan et al. [25] and Mukherjee and Chandra [18].

Now, we consider the initial boundary value problem for velocity $v$, temperature $\Theta$ and strain rates $\nabla v$ as defined below.

Let $u^{i}(x, 0), v^{i}(x, 0)$ and $\Theta^{i}(x, 0)(i=1,2)$ denote the initial displacement, velocity and temperature, respectively. For given density $\rho$, rate of body force $\dot{f}$ in $\Omega_{\mathrm{t}}:=\left(\Omega_{\mathrm{t}}^{1}, \Omega_{\mathrm{t}}^{2}\right)$ and boundary traction $\dot{\mathrm{t}}$ on the Neumann bound$\operatorname{ary} \Gamma_{\mathrm{t}}^{\mathrm{N}}$ we seek $v^{1} \in \Omega_{\mathrm{t}}^{1}, v^{2}$ on $\Gamma_{\mathrm{t}}^{2}:=\Gamma_{\mathrm{tN}}^{2} \cup \Gamma_{\mathrm{tC}}^{2}$ and $\Theta:=\left(\Theta^{1}, \Theta^{2}\right)$ in $\Omega_{\mathrm{t}}$, such that the following system of equations is satisfied

$$
\begin{aligned}
& \int_{\Omega_{\mathrm{t}}^{1}}\left(\nabla v^{1}\right): \mathbb{C}:\left(\nabla \tilde{v}^{1}\right)-\int_{\Omega_{\mathrm{t}}^{1}}\left(\nabla v^{1}\right): \dot{\mathrm{G}}^{\top}(\sigma):\left(\nabla \tilde{v}^{1}\right)+\left\langle S v^{2}, \tilde{v}^{2}\right\rangle_{\Gamma_{\mathrm{t}}^{2}} \\
& +\left\langle\dot{\mathrm{P}}\left(v^{1}, v^{2}\right),[\tilde{v}]\right\rangle_{\Gamma_{\mathrm{tC}}^{1}}-\int_{\Omega_{\mathrm{t}}^{1}} \mathrm{~d}^{\mathrm{n}}: \mathbb{C}: \nabla \tilde{v}^{1}=\int_{\Omega_{\mathrm{t}}^{1}} \rho \dot{\mathrm{f}} \cdot \tilde{v}^{1}+\int_{\Gamma_{\mathrm{t}}^{\mathrm{N}}} \dot{\mathrm{t}} \cdot \tilde{v}^{1} \\
& \int_{\Omega_{\mathrm{t}}}\left[\frac{\partial \Theta}{\partial t} \tilde{\Theta}+\varkappa \nabla \Theta \nabla \tilde{\Theta}\right]-\gamma_{12} \int_{\Gamma_{\mathrm{tC}}^{1}} P \cdot \vec{n}[\Theta][\tilde{\Theta}] \\
& -\int_{\Gamma_{\mathrm{tC}}^{1}} \mu_{\mathrm{f}} \mathrm{P} \cdot \overrightarrow{\mathrm{n}}\left|[v]_{\tau}\right|\left(\gamma_{1} \tilde{\Theta}^{1}+\gamma_{2} \tilde{\Theta}^{2}\right)=0
\end{aligned}
$$

for $\tilde{v}^{1}$ in $\Omega_{\mathrm{t}}^{1}, \tilde{v}^{2}$ on $\Gamma_{\mathrm{t}}^{2}, \tilde{\Theta}$ in $\Omega_{\mathrm{t}}$ and $0 \leq \mathrm{t} \leq \mathrm{T}$.

On the contact boundary $\Gamma_{\mathrm{tC}}^{1}$ the term $\dot{\mathrm{P}}$ denotes the rate of the boundary traction. In the model introduced by Hart [11], the nonelastic part $\mathrm{d}^{\mathfrak{n}}$ of the deformation rate $\mathrm{d}$ describes the viscoplasticity together with the fourth 
order tensor $\mathfrak{G}^{\circ} \mathbb{C}$ denotes the Hooke's tensor, $\mu_{\mathrm{f}}$ the friction coefficient. $\mathrm{S}$ is the Steklov-Poincaré boundary integral operator of linear elasticity (DtN

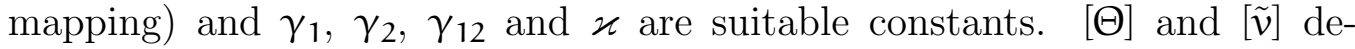
note the jump of the temperature and displacement between the two bodies, respectively. $\vec{n}$ is the exterior normal on $\Omega_{t}^{2}$.

As described by Stephan et al. [25], the heat generation during the process is incorporated by applying a fixed point (staggered) iteration between the equations of mechanical equilibrium and heat conduction. We compute the velocity with finite elements in the work piece and with boundary elements on the boundary of the work tool. The temperature is computed using finite elements in both bodies. The heat conduction equation is discretized with backward Euler in time whereas the viscoplastic material in the work piece is discretized with an explicit Euler time stepping procedure. Finally, the friction at the contact edge between workpiece and tool is computed by a penalty method as performed by Stephan et al. [25].

\section{Conclusion}

As demonstrated the symmetric coupling of finite elements and boundary elements is a powerful approach for solving nonlinear transmission problems (with linear operators in the unbounded region). Optimal convergence rates are obtained when the $\mathrm{hp}$-version together with geometric mesh refinement is used. For the h-version, hierarchical error estimators are cheaper than residual-type error estimators, whereas both estimators lead to similar adaptive refinements. Since the large discrete systems resulting from the FE/BEcoupling are ill-conditioned and dense, preconditioners are crucial for the applicability of iterative solvers. As shown, efficient preconditioners for the $h-, p$ - and hp-versions can be constructed by the Schwarz method. Since in case of linear elasticity, contact problems can be reduced to variational inequalities on the domain boundary, boundary elements can be applied very successfully. Here one can either perform the traditional penalty method or 
implement the more powerful hp-version of the BEM together with the mortar projection. Presented numerical results for adaptive refinements show the strength of the methods. Especially for nonlinear problems like the metal turning process with a viscoplastic working piece and a linear elastic working tool, the FE/BE-coupling gives an efficient solution procedure as described by Denkena et al. [9]. Here, of course, the FEM-part takes care of the viscoplastic material, whereas the linear elastic tool is modelled via boundary elements.

\section{References}

[1] I. Babuška, A. Craig, J. Mandel and J. Pitkäranta. Efficient preconditioning for the p-version finite element method in two dimensions. SIAM J. Numer. Anal. 28 (1991), 624-661. C785

[2] I. Babuška, B. Q. Guo and E. P. Stephan. The hp-version of the boundary element method with geometric mesh on polygonal domains. Computer Methods in Applied Mechanics and Engineering 80 (1990), 319-325. C779

[3] C. Carstensen and E. P. Stephan. Adaptive coupling of boundary elements and finite elements. RAIRO Modl. Math. Anal. Numer. 29 (1995), no. 7, 779-817. C780, C782

[4] A. Chernov. Nonconforming boundary elements and finite elements for interface and contact problems with friction; hp-version for mortar, penalty and Nitsche's methods, PhD Thesis, Universität Hannover, 2007. C789

[5] A. Chernov, M. Maischak and E. P. Stephan. A priori error estimates for hp penalty BEM for contact problems in elasticity. Comput. Methods Appl. Mech. Engrg. 196 (2007), no. 37-40, 3871-3880. doi:10.1016/j.cma.2006.10.044 C788, C789 
[6] A. Chernov, M. Maischak and E. P. Stephan. hp-mortar boundary element methodfor two-body contact problems with friction. Math. Methods Appl. Sci., published online, doi:10.1002/mma.1005 C788, C789, C790

[7] A. Chernov and E. P. Stephan. Adaptive BEM for contact problems with friction. IUTAM Symposium on Computational Methods in Contact Mechanics, 113-122, IUTAM Bookser., 3, Springer, Dordrecht, 2007. C788, C789, C790, C791, C792

[8] M. Costabel and E. P. Stephan. Coupling of finite and boundary element methods for an elastoplastic interface problem. SIAM J. Numer. Anal. 27 (1990), no. 5, 1212-1226. C778

[9] B. Denkena, E. P. Stephan, M. Maischak, D. Heinisch, M. Andres. Numerical computation methods for process-oriented structures in metal chipping. Proceedings of 1st International Conference on Process Machine Interaction (PMI). (2008), 247-258. C776, C795

[10] B. Guo and E. P. Stephan. The hp-version of the coupling of finite element and boundary element methods for transmission problems in polyhedral domains. Numer. Math. 80 (1998), no. 1, 87-107. C779

[11] E. W. Hart. Constitutive relations for the nonelastic deformation of metals, Journal of Enginering Materials and Technology. 98 (1976), 193-202. C793

[12] N. Heuer, F. Leydecker and E. P. Stephan. An iterative substructuring method for the hp-version of the BEM on quasi-uniform triangular meshes, Num.Meth.PDEs. 23 (2007), 879-903. doi:10.1002/num.20259 C787

[13] N. Heuer, M. Maischak and E. P. Stephan. Preconditioned minimum residual iteration for the hp-version of the coupled FEM/BEM with quasi-uniform meshes. Numer. Linear Algebra Appl. 6 (1999), no. 6, 435-456. C783, C784, C785, C786 
[14] N. Heuer and E. P. Stephan. An additive Schwarz method for the h-p version of the boundary element method for hypersingular integral equations in R ${ }^{3}$, IMA J. Numer. Analysis. 21 (2001), 265-283. doi:10.1093/imanum/21.1.265 C787

[15] N. Heuer, E. P. Stephan and T. Tran. Multilevel additive Schwarz method for the hp-version of the Galerkin boundary element method. Math.Comp.. 67 (1998), no. 222, 501-518. C785

[16] M. Maischak and E. P. Stephan. The hp-version of the boundary element method in $\mathrm{R}^{3}$. The basic approximation results. Math. Meth. Appl. Sci. 20 (1997), 461-476. C779

[17] M. Maischak and E. P. Stephan. Adaptive hp-versions of boundary element methods for elastic contact problems, Comp.Mech. 39 (2007), 597-607. doi:10.1007/s00466-006-0109-y C791

[18] S. Mukherjee and A. Chandra. Boundary element formulations for large strain-large deformation problems of plasticity and viscoplasticity, Developments in Boundary Element Methods, 1984, editors P. K. Banerjee and S. Mukherjee, 27-58. C793

[19] P. Mund and E. P. Stephan. Adaptive coupling and fast solution of FEM-BEM equations for parabolic-elliptic interface problems. Math. Methods Appl. Sci. 20 (1997), no. 5, 403-423. C783

[20] P. Mund and E. P. Stephan. An adaptive two-level method for the coupling of nonlinear FEM-BEM equations. SIAM J. Numer. Anal. 36 (1999), no. 4, 1001-1021. C780, C781, C782, C783

[21] P. Seshaiyer and M. Suri. Uniform hp convergence results for the mortar finite element method. Math. Comp. 69 (2000), no. 230, 521-546. C790

[22] E. P. Stephan. Coupling of finite elements and boundary elements for some nonlinear interface problems, Comput. Methods Appl. Mech. Engrg. 101 (1992), no. 1-3, 61-72. C776 
[23] E. P. Stephan. The hp-boundary element method for solving 2- and 3-dimensional problems. Comput. Methods Appl. Mech. Engrg. 133 (1996), no. 3-4, 183-208. C779

[24] E. P. Stephan. Coupling of Boundary Element Methods and Finite Element Methods, Encyclopedia of Computational Mechanics. Vol. 1, Chapter 13. 2004, John Wiley \& Sons. ISBN: 0-470-84699-2. C775, C778

[25] E. P. Stephan, S. Geyn, M. Maischak and M. Andres. A boundary element / finite element procedure for metal chipping. CMM-200\%, June 19-22, 2007, Łódź-Spała, Poland. C793, C794

[26] T. Tran and E. P. Stephan. Additive Schwarz methods for the h-version boundary element method. Applicable Analysis. 60 (1996), no. $1-2,63-84$. C785

[27] T. Tran and E. P. Stephan. Additive Schwarz algorithms for the p-version of the Galerkin boundary element method. Numer. Math.. 85 (2000), no. 3, 433-468. C785

[28] T. Tran and E. P. Stephan. An overlapping additive Schwarz preconditioner for boundary element approximations to the Laplace screen and Lamé crack problems, J. Numer. Math. 12 (2004), 311-330. doi:10.1515/1569395042571265 C787

[29] P. Wriggers. Computational Contact Mechanics, 2002, John Wiley \& Sons. C788, C790

[30] E. Zeidler. Nonlinear Functional Analysis and its Applications. IV. Springer, New York, 1988. C776 


\section{Author address}

1. Ernst P. Stephan, Institute of Applied Mathematics, Leibniz Universität Hannover, GERMANY.

mailto:stephan@ifam.uni-hannover.de 